\title{
Dudas sobre la redacción del derecho territorial
}

\author{
Luz Valle Videla ${ }^{1}$
}

Recibido: 15 de junio de 2017 Aceptado: 10 de julio de 2017

Resumen. Galo Sánchez fue el primero en interesarse por la redacción del derecho territorial castellano. Desde su estudio Para la historia de la redacción del antiguo derecho territorial castellano, se cree que estas colecciones contiene un derecho tosco y rudimentario al presentar muchos capítulos que repiten la misma norma debido a la impericia de sus redactores. Entre las diferentes colecciones, destacaría el Fuero viejo de Castilla al reunir un derecho de carácter más general. Parte de su hipótesis se sustenta en fueros que presentan detalles anecdóticos. Cuando se analizan estos fueros, empero, se descubre que los redactores utilizan la anécdota con unos fines determinados, y esto es lo que se mostrará en este artículo: por un lado, se examinará el objetivo perseguido por los redactores del Libro de los fueros de Castilla y del Fuero viejo al incorporar anécdota al discurso abstracto de los fueros; por otro, se demostrará que este tipo de fuero no se origina a partir de antiguas fazañas.

Palabras clave: redacción del derecho territorial; fueros anecdóticos; fazañas.

\section{[en] Questions regarding the Development of Territorial Law}

Abstract. Galo Sánchez was the first scholar to seriously examine early compilations of Castilian territorial law. Since his study «Para la historia de la redacción del antiguo derecho territorial castellano», it is widely acknowledged that these collections are unpolished, rudimentary legal compilations, as evidenced by the fact that they contain numerous chapters repeating the same laws. Amongst the various collections, the Fuero viejo de Castiella is seen as an exception, since it contains texts of a more general character. Part of Galo Sánchez's hypothesis lays on the fact of fueros displaying anecdotal details. However, when these fueros are analyzed, it becomes clear that the redactors include anecdotal details for specific reasons, as we intend to demonstrate here. This study will examine the aims of the compilers of the Libro de los fueros de Castilla and the Fuero viejo when introducing concrete details into the abstract discourse of the fueros. We will further argue that these fueros did not evolve out of ancient fazañas.

Keywords: elaboration of territorial law; anecdotic fueros; fazañas.

\section{[fr] Doutes sur la rédaction de la loi territoriale}

Résumé. Galo Sánchez a été le premier à s'intéresser à la rédaction de la loi territoriale castillane. Depuis son étude à l'histoire de la rédaction de l'ancien droit territorial castillan, on croit que ces collections sont grossières et rudimentaires, principalement parce que des nombreux chapitres répétent la même norme en raison de l'incompétence des éditeurs. Parmi les différentes collections, je voudrais souligner le Fuero viejo de Castilla, qui rassemble un droit plus général. Une partie de l'hypothèse de Sanchez repose sur des fueros présentant des détails anecdotiques. Cependant, lorsque ces privilèges

Facultad de Letras, Traducción y Comunicación. Universidad Libre de Bruselas

lvallevi@ulb.ac.be 
sont analysés, on y découvre que les rédacteurs ont utilisé l'anecdote à des fins spécifiques. Cet article montrera d'une part, l'objectif poursuivi par les rédacteurs du Livre des privilèges, qui sera celui d'examiner le Fuero Viejo de Castilla en incorporant une anecdote au discours abstrait des fueros; d'autre part, que ce type de juridiction ne provient pas d'anciens fazañas.

Mots clé : rédaction de lois territoriales; anecdotique; fazañas.

Sumario: 1. Introducción. 2. Leyes que se repite y territorialización. 2.1. Galo Sánchez. 2.2. Aquilino Iglesia Ferreirós. 2.3. Gonzalo Oliva Manso. 3. Conclusión. 4. Bibliografía citada.

Cómo citar: L. Valle Videla (2018). «Dudas sobre la redacción del derecho territorial», Cuadernos de Historia del Derecho, XXV, 2018, 11-26.

\section{Introducción}

Cuando se habla de derecho territorial, se hace referencia, sobre todo, a siete textos redactados en Castilla en torno a los siglos XIII y XIV. Estos textos se pueden clasificar según su extensión: el Libro de los fueros de Castiella (LFC) y el Fuero Viejo de Castilla (FVC) son los textos extensos, mientras que Devysas que an les sennores en sus vasallos, el Pseudo ordenamiento de Nájera, el Pseudo ordenamiento de León, el Fuero antiguo de Castilla y el Fuero de los fijosdalgo y las fazañas del fuero de Castilla, los breves.

Quienes más se han interesado por el derecho reunido en estas colecciones y su proceso de redacción han sido los historiadores del derecho, por ejemplo García Gallo o más recientemente, Alvarado Planas². Galo Sánchez en su artículo Para la historia de la redacción del antiguo derecho territorial sentó las bases a partir de las cuales se han desarrollado todos los análisis posteriores ${ }^{3}$. En este explica su formación, su evolución, su desaparición y su filiación mediante el análisis de algunos de los capítulos que componen estas colecciones.

Casi todas ellas ofrecen capítulos introducidos por una frase que anuncia si se trata de un fuero o de una fazaña. La única que no lo hace es las Devysas cuyas reglas aparecen sin ningún tipo de frase introductoria.

Cuando estos historiadores han estudiado el discurso de los fueros y de las fazañas, establecen una clara diferencia entre ambos: los fueros desarrollan un discurso abstracto, en el que no se incluye ningún tipo de dato concreto, mientras que las fazañas describen pleitos e incorporan todo tipo de detalle sobre lo que sucedió en ellos. Si en un mismo capítulo aparecen combinados discurso abstracto y discurso anecdótico, lo achacan a la técnica de los redactores: estos poseían una técnica rudimentaria que los llevó a realizar un trabajo defectuoso e inconsecuente. Para estos estudiosos, en medio de tanta impericia, hay, sin embargo, una obra que destaca por su calidad: el redactor o redactores del FVC supo combinar con mayor pericia las

2 A. García Gallo, «Textos de derecho territorial castellano», Anuario de Historia del Derecho Español, XIII, 1936-1941, pp. 308-396; Javier Alvarado Planas, «El proceso de redacción de los fueros de Castilla», en Historia Iuris: estudios dedicados al profesor Santos M. Coronas González, Oviedo, 2014, pp. 261-278.

3 G. Sánchez, «Para la historia de la redacción del antiguo derecho territorial castellano», AHDE, VI, 1929, pp. 260-328. 
diferentes fuentes utilizadas a la hora de elaborarlo4. Así, se erigiría como la obra mejor acabada del derecho territorial.

Ahora bien, cuando se lleva a cabo una lectura atenta de los capítulos que componen las colecciones territoriales y se analizan los capítulos de discurso abstracto, los de discurso anecdótico y aquéllos que combinan ambos, se descubre que los redactores de este derecho elaboraron las colecciones con una clara conciencia de la importancia de su tarea, intentando crear reglas ricas en matices, con el fin de poder abarcar cualquier problema surgido en una realidad tan compleja como lo es la de Castilla en los siglos XIII y XIV. Estos redactores fueron capaces de reunir numerosos capítulos que trataban temas similares para abordar un mismo tipo de conflicto a partir de diferentes perspectivas. En este contexto, la anécdota adquiere un valor fundamental, dado que posee una función clara, al permitir aproximar las reglas de una experiencia concreta.

Para mostrar esto, volveré a examinar el análisis realizado por los historiadores del derecho. Es imprescindible repasar los ejemplos que dan para identificar los problemas que surgen cuando analizan el discurso de las diferentes reglas que conforman las colecciones de derecho territorial. Esto me permitirá dar otra explicación del uso de la anécdota en estas colecciones. Debido a que todos los capítulos de las colecciones breves aparecen en el FVC, en este artículo solo analizaré las dos colecciones extensas.

\section{Leyes que se repiten y territorialización}

Los historiadores del derecho han estudiado la técnica de los diferentes redactores del derecho territorial para tratar de comprender la materia a partir de la cual elaboraron sus colecciones y han llegado a la conclusión de que están plagadas de repeticiones. No obstante, habría una colección que sobresaldría al estar redactada con mayor esmero: se trataría del FVC, cuyos capítulos tendrían un mayor alcance territorial. Al ser Galo Sánchez quien sentó las bases de esta visión de la redacción de este derecho 5 , examinaré en detalle su estudio, aunque también tendré en cuenta a aquellos historiadores que han añadido elementos nuevos a la teoría de este último, es decir, Aquilino Iglesias Ferreirós y Gonzalo Oliva Manso ${ }^{6}$.

\subsection{Galo Sánchez}

Galo Sánchez analizó en detalle la técnica utilizada por los redactores de las colecciones territoriales. Tras su estudio, llegó a la conclusión de que, tanto en el LFC como en el FVC, es muy común encontrar dos o tres capítulos en los cuales una misma ley se repite. También vio que los autores del LFC y del FVC utilizaron una misma fuente cuando elaboraron sus colecciones, al compartir numerosos fueros y fazañas, pero que, a la hora de manejarla, el redactor del FVC fue mucho más hábil de lo que lo fue el del LFC. Estas conclusiones las sustenta en el comportamiento de

J. Alvarado Planas y G. Oliva Manso, Los fueros de Castilla, Madrid, 2004, p. 199.

G. Sánchez, «Para la historia de la redacción del antiguo derecho territorial castellano», AHDE, VI, 1929.

A. Iglesias Ferreirós, «Derecho municipal, derecho señorial, derecho regio», Historia, Instituciones, Documentos, IV, 1977; J. Alvarado Planas y G. Oliva Manso, Los fueros de Castilla, Madrid, 2004. 
ciertos capítulos, sin embargo, cuando se analizan estos capítulos, se ven contradicciones importantes.

Empecemos por las repeticiones. Según Galo Sánchez recidos, se debe a que se han incorporado diferentes versiones de la misma norma: en estas colecciones encontraríamos la versión original, además de versiones posteriores, que estarían mejor redactadas y serían las que permitirían comprender el contenido de la norma. Galo Sánchez distinguió dos tipos de repetición. La primera es la que se ve en los capítulos 34, 61 y 249 del LFC ${ }^{8}$. La segunda es la que se ve cuando los fueros incorporan discurso anecdótico.

Empiezo por el primer tipo. Obsérvense los tres capítulos que Sánchez propone como muestra de repetición:

34

Esto es por fuero : que sy judio demanda por carta alguna deuda e non la puede prouar, deue tener el alcalle la otra. Et si lo pvdiere prouar, que aya su deuda; e peche el que niega sesenta sueldos. Et sy non lo pudiere prouar commo lo dise la carta, sea quita la deuda; e sesenta sueldos el jvdio. Et sy el escriuano que fiso la carta testiguare con otro jvdio, non cumpla ; que sin el escriuano que fiso la carta deue prouar con judio e con cristiano.
61

Esto es por fuero : que sy judio demanda a cristiano deuda por carta et dise el cristiano que non le a de dar nada, deue el alcalle tomar la carta, et deue sacar los pesquiridores. Et sy el judio pudier prouar, deuel dar la deuda el cristiano ; et peche sesenta sueldos. Et sy judio non prouar la carta, deue perder la deuda e pechar sesenta sueldos.
249

Esto es por fasannya : que demandaua Mose Amordosiel de Burgos deuda por carta a Ferrant Yuannes, fijo de don Pascoal ; e Johan Pascoal era muerto ; et en aquella carta por que demandaua el judio a Ferrant Yuannes por su padre, e leyeron la carta ante el alcalle, e dixo Ferrant Yuannes que fijo era de muerto, e que prouas el judio a Ferrant Yuanes la carta e la deuda commo era derecho e quela deuda pagaria commo fuere derecho. Et dixo el judio que prouado lo auya ; e tomo la carta el alcalle e dixo Ferrant Yuannes al alcalle que non diesse la carta, que sy non pudiesse prouar la carta el judio deue perder la deuda de la carta e pechar sesenta sueldos, pues rasono ante el alcalle que prouada auya la carta. Et sy non pudiesse prouar commo auya prouado la carta, jusgo el alcalle que prouasse el judio la carta dela deuda con judio e con cristiano, que por que dixo el judio que prouada auya la carta non deuya perder la deuda ; e que cogiesse su deuda. Et el otro que era fijo de muerto diesse la deuda prouada e non pechasse sesenta sueldos al meryno. Et sy el judio non prouasse la carta, que perdiesse toda la deuda de la carta e non pechasse al meryno, por que era la demanda de omne muerto.

El fuero 34 y el fuero 61 contendrían la misma ley. El fuero 34 representaría la ley primitiva y el fuero 61, la versión más moderna. Galo Sánchez cita, además, la fazaña 249 como ejemplo de capítulo que repite la misma ley evocada en los fueros 34 y 61 , sin especificar de qué versión se trata. A pesar de esto, se puede deducir gracias a

G. Sánchez, «Para la historia de la redacción del antiguo derecho territorial castellano», AHDE, VI, 1929, pp. 275-276.

8 Como mi estudio se basa en el análisis llevado a cabo por Galo Sánchez, examinaré el comportamiento del discurso de los capítulos en su edición del LFC: G. Sánchez, El libro de los fueros de Castiella, Barcelona, [1924] 1981. 
lo que dice sobre la dependencia entre fueros y fazañas', que la fazaña supone el origen de los dos fueros, es decir que estaríamos ante la versión más arcaica de los tres capítulos. Ahora bien, cuando se analizan en detalle estos ejemplos, se descubre que, en realidad, cada uno de los capítulos trata un aspecto diferente del mismo conflicto jurídico. Esto se puede comprobar si se tienen en cuenta lo siguiente:

1. Si los fueros 34 y 61 describen el requerimiento por carta de un hombre judío, la fazaña 249 describe el requerimiento por carta de un judío burgalés. El hecho de que en la fazaña se especifique que el requerimiento lo realiza alguien presentado mediante un topónimo indica que se trata del requerimiento de un extranjero, ajeno a la comunidad donde lo hace ${ }^{10}$. En la legislación medieval, el hecho de ser extranjero, nuevo residente o ciudadano de una ciudad o villa confería estatus jurídicos diferentes, además de derechos diferentes. Los fueros intentaban legislar teniendo en cuenta la relación que mantenían las personas con el territorio en el cual surgía el conflicto $^{11}$. Es importante, por tanto, darse cuenta de que la fazaña 249 es diferente de los dos fueros, porque evoca un pleito en el cual la demanda es hecha por un hombre extranjero que, además, es de religión judía.

2. El problema jurídico planteado en el fuero 34 y en el fuero 61 es el mismo, sin embargo, el caso que se trata en la fazaña, no: en los fueros se trata de una demanda de reembolso tratada por carta, mientras que en la fazaña se expone un caso de deuda contraída por un muerto.

3. La fazaña habla de un hombre judío que ya había establecido la existencia de la deuda una primera vez y que debe probarlo una segunda vez. Por su parte los fueros contemplan la posibilidad de que un hombre judío pruebe la deuda una sola vez.

4. El alcalde que aparece en la fazaña encuentra una solución al conflicto y dictamina que ninguna de las partes pague la multa al merino, independientemente de que la deuda se pruebe o no. Los fueros en cambio, imponen una multa a la parte culpable que deberá pagar al merino.

5. Los problemas jurídicos abordados en los dos fueros presentan matices que obligan a diferenciarlos. El fuero 34 parte de la base de que el hombre judío que reclama la deuda no la puede probar, pero como dicha deuda figura en la carta, este puede acudir o bien a un escribano o bien a dos testigos para demostrarla. Esta es la razón por la cual hay un planteamiento inicial diferente: el alcalde debe tener la otra carta porque en esta se muestra que la deuda existe. El fuero 61, en cambio, trata de una deuda por carta entre un hombre judío y un hombre cristiano, pero en este caso se da por sentado que el primero no prueba la carta.

6. En la fazaña se ve con claridad que tanto el alcalde como Fernando Ibáñez, una de las partes que intervienen en la fazaña, repiten el contenido del fuero 34. Si nos fijamos primero en Fernando Ibáñez, vemos que este cita una parte del fuero 34:

G. Sánchez, «Para la historia de la redacción del antiguo derecho territorial castellano», AHDE, VI, 1929, p. 267.

10 Francisco Ruiz Gómez ha analizado la presentación de los extranjeros mediante topónimos en la villa de Oña (Las aldeas castellanas en la Edad Media. Oña en los siglos XIV y XV, Madrid, 1990, pp. 29-31). Ver igualmente mi artículo «Antroponimia y representación en el Libro de los fueros de Castiella», en C. García de Lucas y A. Oddo (ed.), Magister dixit. Mélanges offerts à Bernard Darbord, París, 2016, pp. 419-433.

11 A. García Ulecía, Los factores de diferenciación entre las personas en los fueros de la Extremadura castellano-aragonesa, Sevilla, 1975, pp. 41-64; P. Domínguez Lozano, Las circunstancias personales determinantes de la vinculación con el derecho local. Estudio sobre el derecho altomedieval y el derecho local de Aragón, Navarra y Cataluña (siglos IX-XV), Madrid, 1988, pp. 39-54. 
[...] non pudiesse prouar la carta el judio deue perder la deuda de la carta e pechar sesenta sueldos [...]. Esta afirmación muestra que este hombre bueno conocía el fuero. Pasa lo mismo con el alcalde: [...] jusgo el alcalle que prouasse el judio la carta dela deuda con judio e con cristiano [...]. El hecho de que los dos acudan a la misma norma y la citen muestra de manera clara que el fuero 34 es anterior a la fazaña. La otra posibilidad, la de contemplar que la fazaña es anterior al fuero, implica un proceso de creación mucho más complicado y menos plausible: ambos habrían creado una norma en un juicio, a partir de la cual surgiría en primer lugar el fuero 34, para después crear el fuero 61.

El hecho de que haya tantas diferencias entre los capítulos constituye la prueba de que el autor no repite las leyes por falta de maña o por tener una técnica rudimentaria $^{12}$. Por el contrario, lo que hace es completar y matizar las leyes en función de las diferentes posibilidades que contempla: las diferentes versiones se completan y proporcionan reglas más exhaustivas, mejor adaptadas a las diferentes variantes que podrían presentarse a la hora de tener que elucidar un juicio ${ }^{13}$.

Pero, ¿qué nos dice Galo Sánchez sobre este tipo de repetición en el FVC ${ }^{14}$ ? En este caso se muestra mucho más reservado. Cuando aborda el tema, casi no habla de que haya problemas de repetición. Sólo en una ocasión cita capítulos anecdóticos de esta colección como ejemplo. Para él, los capítulos 1.2.4 y 1.6.4 son repeticiones de la misma ley y explica que se produce porque el redactor fue incapaz de sintetizarlos en un solo capítulo debido a su impericia ${ }^{15}$. Sin embargo, cuando se analizan, se constata que el fenómeno es muy similar a lo que ocurre cuando se comparan los fueros 34 y 61 del LFC. Los dos capítulos del FVC tratan de la venta de vino en un palacio, empero, entre ambos hay una diferencia importante: en el primer caso se trata de una venta en un palacio real, en el segundo, el palacio es de un noble. Es cierto que estos dos capítulos tratan el mismo tema, pero el sector de la población afectado por la regla varía de uno a otro. No hay que confundir cercanía e identidad $\mathrm{y}$, desde mi punto de vista, la presencia de los dos capítulos no es de ninguna manera una prueba de impericia por parte del redactor. Al contrario, nos muestra su determinación de enriquecer la colección volviéndola exhaustiva. Si ha incluido dos normas diferentes, es porque quería tener en cuenta dos destinatarios diferentes y de ahí que los haya hecho aparecer en dos apartados diferentes de la colección. En efecto, el FVC se organiza en libros que a su vez se dividen en títulos y estos en leyes. El redactor sitúo la norma sobre el palacio real en el título dedicado al rey (1.2.4) y, la norma dedicada a la nobleza en el título dedicado a los fijosdalgo (1.6.4).

El segundo tipo de repetición del cual habla este historiador se puede ver en los capítulos que presentan una combinación de discurso abstracto y discurso anecdóti-

12 Galo Sánchez presenta como repeticiones los capítulos 2 y 55, 167 y 278, 133 y 134, 142 y 192 (G. Sánchez, «Para la historia de la redacción del antiguo derecho territorial castellano», $A H D E$, VI, 1929, p. 275). Sin embargo, cuando se analiza en profundidad su contenido, se observa que no lo son.

13 Johannes Kabatek también ha reflexionado sobre este tema y cree que el LFC proviene de un círculo de personas cultas y eruditas. Desde su punto de vista, escribir el texto en castellano implicaría, además, que el redactor quería legitimar su derecho y su historia (Die Bolognesische Renaissance und der Ausbau romanischer Sprachen. Juristische Diskurstraditionen und Sprachentwicklung in Südfrankreich und Spanien im 12. und 13. Jahrhundert, Tübingen, 2005, p. 223).

14 Para analizar los textos del FVC, utilizaré la edición realizada por Benjamín González Alonso, Ángel Barrios García y Gregorio del Ser Quijano (Fuero viejo de Castilla. Consideraciones sobre la historia del derecho de Castilla (c. 800-1356), Valladolid, 1996).

15 G. Sánchez, «Para la historia de la redacción del antiguo derecho territorial castellano», AHDE, VI, 1929, p. 288. 
co. Para él, ambas partes tienen el mismo contenido, pero con dos formas diferentes: la norma precede o sigue al resumen del proceso que la ha motivado ${ }^{16}$. Los redactores de los fueros deberían haber suprimido todos los detalles anecdóticos de las fazañas con las cuales trabajaban, hasta transformar el pleito en una regla abstracta, pero, dado que eran demasiado inexpertos, descuidaron su labor y crearon capítulos en que ambos discursos aparecen mezclados. Como ejemplos cita los capítulos 2 y 11 del LFC. No obstante, cuando se analiza el capítulo 2, se ve con claridad que no hay una combinación de discursos, sino que se trata de un capítulo exclusivamente anecdótico. ¿Por qué lo clasifica como un fuero que combina ambos discursos si sólo hay anécdota? ¿Estamos ante una errata? En todo caso, el capítulo 3 sí muestra dicha estructura: en un primer momento, se enuncia una norma y a continuación aparece un pleito relacionado con la norma. Pasa lo mismo con el capítulo 11.

Ahora bien, además de estos dos capítulos, me gustaría subrayar que este tipo de discurso se da con frecuencia no sólo en el LFC, sino también en el FVC. En el LFC, en los capítulos $23,25,74,88,106,133,142,149$ y 181 vemos que la norma precede a la anécdota y en los capítulos 116, 151,206, 232, 284, 290, en primer lugar aparece la anécdota y a continuación la norma.

En el FVC los capítulos 1.2.2, 1.2.4, 1.3.2, 1.5.5, 1.5.10, 1.6.4, 3.1.7, 3.1.8 y 4.2.3 responden al primer patrón, en cambio no se ha incluido ninguno que responda al segundo.

Si se analiza la forma que los redactores dan al discurso, haciendo preceder o seguir la anécdota a la norma, conjuntamente con la manera de nombrarlos, se observa algo muy llamativo: los redactores los nombran atendiendo a una pauta. En el primer caso, nos enfrentamos a fueros y en el segundo, a fazañas. Hay tres excepciones: en el LFC, el capítulo 3 no tiene ningún tipo de frase introductoria que especifique si se trata de un fuero o de una fazaña y, en el FVC, el capítulo 1.5.5 es presentado mediante la combinación de ambos términos: Esta es la fazanna de fuero de Castiella. Por otro lado, en cuanto al tipo de discurso, el fuero 151 sería un fuero más del LFC de no ser porque, al principio, se nos informa de que quien juzgó fue el rey Fernando. Debido al marco de mi estudio y lo complejo del tema, solo hablaré de los capítulos en los que el compilador presenta primero la norma y a continuación la anécdota.

Cuando se analizan en su conjunto estos capítulos, se ve que los redactores utilizan la anécdota tras la norma abstracta con diferentes fines y la relación entre la parte abstracta y la parte anecdótica varía de unos capítulos a otros. Veámoslo a continuación:

1. Lo más llamativo al analizarlos conjuntamente, es que sólo en algunos fueros puede relacionarse la anécdota con el origen de la norma. Se trata de dos capítulos en los que el rey juzga (FVC: 1.2.4, 1.6.4). Sin embargo, el hecho de que se cite al rey como fuente de la norma podría señalar en otra dirección, al igual que ocurre en los otros fueros, y es que, más allá del origen de la norma, citar a esta autoridad podría estar relacionado con el hecho de que el redactor quiso legitimarla ${ }^{17}$ : el rey fue quien juzgó, la norma es, por lo tanto, válida.

2. En otros casos (LFC: 23, 74, 88, 133; FVC: 3.1.8, 4.2.3), cuando se examina la anécdota y se compara con la norma, no está tan claro que aquella sea la versión

Ibidem, p. 275.

17 Para los procedimientos de legitimación de un discurso ver P. Charaudeau y D. Maingueneau, Dictionnaire d'analyse du discours, París, 2002, p. 86. 
primitiva a partir de la cual se realizó esta. Por lo general, los datos que el redactor incluye en la parte anecdótica son muy escasos y ninguno de ellos lo prueba. Por el contrario, como en todos los capítulos se da el nombre de las partes o de la autoridad que interviene en los pleitos, cuando he encontrado un testimonio histórico de su existencia, he descubierto que estas personas son contemporáneas de los redactores, algo que excluye la posibilidad de que la norma provenga de un pleito que tuvo lugar en una época anterior a la redacción de las colecciones ${ }^{18}$. Entonces, ¿por qué el redactor incorpora el nombre de las partes al final de los fueros? Podría ser, una vez más, otra técnica empleada por el redactor para dar mayor autoridad a su discurso. Este pudo incluir al final de los fueros ciertos nombres clave para corroborar lo expuesto en la norma, puesto que citar a los participantes en un juicio recordaría lo que pasó, además de demostrar que la norma ya se había utilizado para juzgar un pleito. El hecho de nombrar las partes o la autoridad bastaría para crear un diálogo implícito con sus interlocutores. El redactor eligió casos prácticos conocidos al formar parte de la memoria colectiva reciente del lugar en que fueron juzgados ${ }^{19}$. Sobre el tema de dar credibilidad a un discurso, es muy interesante la reflexión llevada a cabo por Marie-Claude Malenfant cuando analiza la importancia que daba Quintiliano (\$41) tanto a lo dicho como a lo hecho por un juez, por una de las partes o por el abogado de estas para persuadir a un auditorio ${ }^{20}$ :

Cette dernière catégorie, qui peut sembler étrangère à l'exemplum, s'inscrit pourtant rigoureusement parmi ses espèces puisque l'orateur donnera du crédit au point dont il veut persuader son auditoire par similitude avec un dit ou un fait déjà connus; or les paroles ou les actes des membres du procès sont pour ainsi dire immédiatement connus des auditeurs car ils sont contemporains du moment où la cause est entendue.

3. Otros muchos fueros, además de indicar el nombre de las partes al final, incluyen detalles sobre los pleitos que los erigen en verdaderos ejemplos. En estos, el redactor incluye información que va más allá de la simple regla enunciada al principio del capítulo, y su voz adquiere un tono didáctico: en unos casos insiste en cómo las partes, tras la sentencia, la ejecutan sin rechistar (LFC: 25, 149; FVC: 1.3.2, 1.5.5, 3.1.7); a veces, da un contraejemplo que informa de las nefastas consecuencias que acarrea no respetar la norma (LFC: 106) e incluso, al dar el contraejemplo (LFC: 142), el redactor aprovecha para añadir, a partir de este, una enseñanza jurídica. En definitiva, el redactor adopta un tono didáctico y utiliza la anécdota para convencer a su público de la legitimidad del contenido de las normas expuestas. Se podría ver en estos fueros un récit bref donné comme veridique et destiné à être inséré dans un discours [...] pour convaincre un auditoire par une leçon salutaire, definición dada por Claude Bremond y Jacques Le Goff de lo que es un exemplum ${ }^{21}$.

18 L. Valle Videla, «El discurso referido en los fueros anecdóticos del Libro de los fueros de Castiella», en L. Valle Videla, El discurso referido en los textos medievales españoles, París, 2013, pp. 159-161.

19 Este tema lo he abordado al estudiar la relación entre el LFC y la villa de Belorado (L.Valle Videla, «Los elementos concejiles en los capítulos anecdóticos del Libro de los fueros de Castiella», en J. Alvarado Planas, El municipio medieval: nuevas perspectivas, Madrid, 2009, pp. 201-219).

20 M. C. Malenfant, Argumentaires de l'une et l'autre espèce de femme. Le statut de l'exemplum dans les discours littéraires sur la femme (1500-1550), Quebec, 2003, p. 37.

21 C. Bremond, J. Le Goff y J. C. Schmitt, L'exemplum, Turnhout, 1996, pp. 37-38. 
4. Dentro del grupo de los fueros que incorporan anécdota al final, cuatro merecen una atención particular, porque contradicen de lleno la hipótesis de Galo Sánchez. Los cuatro incorporan elementos lingüísticos que ponen en entredicho que la anécdota suponga el origen de la norma. Hasta ahora, los fueros analizados han incorporado la anécdota mediante el verbo juzgar tras la regla abstracta. Este verbo funciona en estos casos como introductor de discurso referido y alude al origen jurídico de la anécdota, conectándola directamente con el fuero que aparece al principio $^{22}$. Sin embargo, en tres de los fueros anecdóticos (LFC: 3, 181; FVC: 1.5.10), los redactores no utilizan este verbo, sino el verbo contecer. Iniciar la anécdota con este verbo le proporciona un significado diferente, por cuanto al utilizarlo el redactor insiste en que los hechos evocados han acaecido y esto es lo importante para él. La anécdota se reivindica como histórica, se da por cierta y así nos lo señala desde el principio. Además, cuando los redactores usan este verbo, introducen información en la parte anecdótica sobre aspectos de los enfrentamientos que poco tienen que ver con el juicio en sí mismo. Mediante esta técnica, lo que hacen es dirigir la atención del lector hacia lo ocurrido antes del pleito y dejar en un segundo plano el juicio: en el capítulo 3, se nos cuenta que un señor, que ha forzado a una de las mancebas que le sirven, huye de la villa para refugiarse en casa del rey y, en el fuero 181 del LFC así como en el capítulo 1.5.10 del FVC, que un señor quiere proteger a sus vasallos de una enemistad surgida a raíz de sus actos. Los redactores dan tanta importancia a los hechos que rodean al juicio como al juicio en sí mismo e incluyen las sentencias al final, sentencias que emparentan la anécdota con la norma del principio. Una vez más, vemos, gracias a estos elementos, que la anécdota incorporada al fuero, no es una mera repetición que debió suprimirse, sino que adquiere vida propia y nutre el discurso de los fueros. Hace que estos se vuelvan más persuasivos, más reales y se aproximen con mayor facilidad a la experiencia del auditorio al que iban dirigidos.

Por último, me queda por analizar dentro de este grupo, el capítulo 1.2.2 del FVC. Este capítulo, según me parece, es el capítulo más problemático para la hipótesis de Galo Sánchez. En él, el redactor dice explícitamente [...] E esto fue judgado por muchos rricos omes en Castiella. Et después fue judgado por Rruy Sánchez de Navarra [...]. Gracias al inicio de la anécdota, observamos que a pesar de que la norma se ha utilizado en numerosas ocasiones para juzgar a muchos ricos hombres, el redactor decide hablar en este fuero de la vez en que se usó para juzgar a Sánchez de Navarra. Ilustra la norma con un pleito pertinente por su contenido y no por tratarse del primero en que supuestamente esta se utilizó. Con él trata de persuadir a su auditorio de que la norma expuesta es legítima. El hecho de que se ilustre la norma con lo que le ocurrió a este hombre contradice de lleno la evolución propuesta por Galo Sánchez. Este ejemplo obliga a revisar el funcionamiento de la anécdota en los fueros y la relación que se ha establecido hasta ahora entre fuero y fazaña, dado que la anécdota de los fueros no es un vestigio de un pleito ocurrido en una época anterior. Sin quererlo, Sánchez de Navarra se ha transformado en un elemento fundamental, no sólo para ilustrar esta norma, sino también para comprender mejor por qué en los fueros se incluye discurso anecdótico.

Debido a todos estos aspectos, es inadecuado postular, por tanto, que los redactores de estas colecciones eran torpes e incapaces de llevar a cabo una refundición adecuada de las fuentes. Estos redactores dan muestras de perseguir un objetivo con-

L. Valle Videla, «El discurso referido en los fueros anecdóticos del Libro de los fueros de Castiella», en L. Valle Videla, El discurso referido en los textos medievales españoles, París, 2013, pp. 162-166. 
creto cuando incorporan anécdota en los fueros. Si bien es cierto, no son sistemáticos, pero ¿acaso en los siglos XIII y XIV se puede juzgar la calidad de un discurso a partir de este criterio?

Para terminar con el análisis de Galo Sánchez sobre la técnica de redacción de los compiladores del derecho territorial, es necesario hablar del fenómeno de la territorialización ${ }^{23}$. Según este investigador, al comparar los capítulos que comparten el FVC y el LFC, se ve de manera clara que el primero está mucho mejor redactado, porque numerosas leyes locales se han transformado en leyes de aplicación más general. Para justificar esta afirmación, compara los siguientes capítulos anecdóticos ${ }^{24}$ :

$\begin{array}{ll}\text { LFC } & \text { FVC } \\ 25 & 3.4 .7 \\ 186 & 5.6 .2 \\ 211 & 2.1 .4 \\ 271 & 1.5 .14\end{array}$

Al revisar los ejemplos que este estudioso facilita cuando compara ambas colecciones, se observa que su análisis resulta insuficiente para afirmar que el FVC contiene un derecho de carácter más general que el LFC. La teoría de la territorialización no justifica las modificaciones introducidas en ambas colecciones, puesto que se puede ver, por un lado, que los capítulos 25 y 3.4 .7 no son los mismos; por otro, que en los capítulos 186 y 5.6.2, 211 y 2.1.4, 271 y 1.5.14 la supresión de detalles superfluos en el FVC no ha tenido lugar. Detengámonos en los capítulos 186 y 5.6.2. Para facilitar la comparación destaco en negrita las partes más significativas.

\section{LFC 186}

Esto es por fasannya : que Lope Gunçales de Sagrero e sus fijos de donna Mariscote demandauan partiçion adon Rodrigo de Sagrero su tio e a Ferrant Romero et adona Eluyra de Cubo que les diesse partiçion de dona Rama su tia. Et dieron les a partir en una heredat e despues non querian dar les a partir en lo al por que eran fijos de barraganna. Et jusgaron les los adelantados por fuero que pues dado les auyan apartir en una heredad, quela partiçion yr deuia adelante ; e oyeron les de dar en todo apartir.

\section{FVC 5.6.2}

Esto es fuero de Castiella. Que Lope Goçález de Segrero e sus parientes, fijos de don Mariscote, demandavan partiçión a don Rrodrigo, su tío, e a Ferrant Rromero e a donna Elvira de Cubo, que les diesen partiçión de la buena de donna Rrama, su tía, que fuera monja ; e diéronles a partir en la una heredat e después non les querién dar a partir en los otros bienes de aquella su tía, porque eran fijos de barragana. Et judgaron los alcalles por fuero que, pues dádoles avién a partir en la una heredat, que la partiçión yr devié adelante; e asy oviéronles a dar a partir en todo.

Gracias a la comparación de estos dos capítulos, puedo realizar los siguientes comentarios:

1. Las dos colecciones presentan textos muy similares. Como los cambios que el autor del FVC introduce en su capítulo son insignificantes, la ley no resulta ni más general ni presenta un mayor carácter territorial.

\footnotetext{
23 G. Sánchez, «Para la historia de la redacción del antiguo derecho territorial castellano», AHDE, VI, 1929, pp. 297-307.

24 Ibidem, p. 300.
} 
2. Si se debe aceptar la hipótesis de que uno de los dos autores introduce detalles inútiles, entonces este es el autor del FVC, puesto que da información superflua sobre la persona que ha dejado la herencia. Así, el hecho de que doña Rama sea buena o mala no tiene ninguna incidencia en la sentencia, como nos muestra el LFC.

3. Si el autor del FVC prefiere utilizar una frase para decir que la familia de Lope Sagrero no quería compartir con él sus otros bienes: [...] e diéronles a partir en la una heredat e después non les querién dar a partir en los otros bienes de aquella su tía [...], el autor del LFC no resulta menos exhaustivo cuando utiliza el pronombre al: [...] Et dieron les a partir en una heredat e despues non querian dar les a partir en lo al $[\ldots]^{25}$. Esta frase muestra que los dos autores utilizan diferentes medios lingüísticos, para expresar el mismo contenido.

La territorialización defendida por Galo Sánchez no se corresponde, por tanto, con los cambios introducidos en estos dos capítulos.

Con respecto a los capítulos 25 y 3.4.7, se constata que ambos tratan un problema de deuda entre dos hombres. Según Galo Sánchez, se han eliminado del fuero 3.4.7 todos los detalles anecdóticos mediante el proceso de la territorialización, mientras que el capítulo 25 del LFC los ha conservado al final. Empero, cuando se leen los dos capítulos, se descubre que se trata de dos tipos de deuda diferente: en el capítulo 25, el compilador aborda un caso en el que el deudor está enfermo o bien de gota o bien con fiebre. Además de las precisiones que aporta sobre las circunstancias de un pleito en tales casos, el redactor contempla la posibilidad de que alguien tenga que jurar en un pleito por partición fuera de su villa. Ahora bien, jurar en un proceso no es lo mismo que deber dinero. El capítulo 3.4.7 del FVC tiene en cuenta dos aspectos diferentes de la deuda al considerar la posibilidad de que el acreedor vaya a la ciudad del deudor, aunque este último estuviera postrado en la cama, pero sin realizar los trámites necesarios para que el deudor le devuelva su dinero. Además, contempla la posibilidad de lo que deben hacer los hijos del deudor en caso de que este muera y el acreedor no haya actuado cuando el padre seguía vivo. Una vez más, nos enfrentamos a dos capítulos que tratan problemas desde ángulos distintos y no a dos maneras diferentes de redactar la misma regla. Pasa lo mismo si se compara el capítulo 211 con su homólogo, el capítulo 2.1.4 del FVC, así como cuando se comparan los capítulos 271 y 1.5.14. El autor modifica en algunos casos la frase con la que el autor inicia los capítulos, sin embargo esto no es bastante para afirmar que ha habido una territorialización de la ley.

La debilidad de la hipótesis de Galo Sánchez se acrecienta si se analizan sus comentarios sobre los capítulos 4.6.1 del FVC y 46 del LFC ${ }^{26}$. Tras la comparación concluye que el autor del FVC ha producido una redacción más cuidada que la producida por el autor del LFC, dado que al redactar su capítulo evoca todos los datos y todos los detalles del pleito porque trata de dar una versión más exhaustiva. El redactor del LFC ofrecería la versión más cercana a la fuente y, por tanto, más descuidada.

Cuando se revisa esta comparación, se observa, sin embargo, que el autor del LFC lleva a cabo un proceso de simplificación, sin descuidar por ello el contenido del pleito, lo que lo vuelve más sintético. Puesto que todos los aspectos esenciales

25 Su significado es otra cosa (J. Corominas y J. A. Pascual, Diccionario crítico etimológico castellano e hispánico, Madrid, 1980, vol. I, p. 99).

26 G. Sánchez, «Para la historia de la redacción del antiguo derecho territorial castellano», $A H D E$, VI, 1929, pp. 298-300. 
se han conservado, ¿no es legítimo preguntarse en este caso si quien ha aplicado el proceso de territorialización es el redactor del LFC? En efecto, este ha tomado la anécdota y la ha despojado de muchos detalles innecesarios, lo que provoca que el capítulo resulte más informativo y mucho menos descriptivo de lo que resulta el capítulo del FVC. Asimismo, la frase introductoria de uno y otro son diferentes: si en el LFC se nos dice que estamos ante un fuero, en el FVC no se incluye ninguna frase al principio que permita saber si se trata de una fazaña o un fuero. A pesar de todo esto, Galo Sánchez no contempla esta posibilidad, porque para él el trabajo del redactor del LFC es simplemente inferior, así afirma categórico: Nunca ocurre lo contrario, esto es, que el libro haya territorializado un precepto local de X y el Fuero viejo no ${ }^{27}$.

Galo Sánchez no analiza la anécdota de manera sistemática y como consecuencia llega a conclusiones erróneas. Los redactores no repiten la anécdota inútilmente. Para estos, cumple una función esencial dentro de las colecciones. Además, el fenómeno de la territorialización no explica la diferencia entre el discurso de los capítulos compartidos por estas dos colecciones. Su teoría, sin embargo, sentó las bases de todos los análisis posteriores sobre la redacción de este tipo de derecho y es así como se ha llegado a la falsa creencia sumamente extendida de que cuando un fuero presenta anécdota se debe a que procede de una antigua fazaña ${ }^{28}$.

\subsection{Aquilino Iglesia Ferreirós}

Aquilino Iglesias es otro historiador del derecho que también se ha interesado por estas colecciones. A pesar de que no estudia en profundidad su proceso de redacción, critica la hipótesis de la territorialización defendida por Galo Sánchez ${ }^{29}$. Para Iglesias, los capítulos compartidos por el FVC y el LFC no se sometieron a tal proceso, cuando se incorporaron al primero. Para demostrarlo, menciona numerosos capítulos, pero sólo algunos de ellos presentan discurso anecdótico. Para él, los fueros 25, 46, 88 y 150 del LFC no se territorializaron cuando se incorporaron al FVC, respectivamente 3.4.7, 4.6.1, 3.6.5 y 4.6.6.

Sin embargo, para rebatir la hipótesis de Sánchez no analiza ni la composición de los capítulos ni su discurso, sino que se contenta con sacar conclusiones a partir de la frase que los introduce. Así, no explica por qué el fuero 88 del LFC incorpora discurso anecdótico al final y el fuero 3.6.5 del FVC no. Tampoco dice nada sobre por qué el fuero 46 es mucho más sintético que el capítulo 4.6.1. Debido a esto, su crítica resulta poco útil para valorar y comprender la tarea hecha por los redactores de estas dos colecciones.

\subsection{Gonzalo Oliva Manso}

En 2004, Javier Alvarado Planas y Gonzalo Oliva Manso publicaron una edición de las colecciones de derecho territorial. A esta edición, le precede un estudio crítico en el cual analizan, entre otros aspectos, la formación del derecho territorial siguiendo

\footnotetext{
Ibidem, p. 301.

28 J. Kabatek, «Tradiciones discursivas y cambios lingüísticos», Lexis. Revista de lingüistica y literatura, XXIX/2, 2005, p. 161.

29 A. Iglesia Ferreirós, «Derecho municipal, derecho señorial, derecho regio», Historia, Instituciones, Documentos, IV, 1977, p. 147, nota 146.
} 
las pautas de Galo Sánchez; empero, solo Oliva Manso se interesa por los capítulos anecdóticos. Como mi objetivo es comprender mejor cómo utilizan los redactores de las colecciones la anécdota, solo revisaré las hipótesis de este historiador.

Cuando Oliva Manso aborda el tema de cómo fueron redactadas estas colecciones, sólo presta atención a la hipótesis de la territorialización y, además de los capítulos propuestos por Galo Sánchez, propone otros para confirmar este proceso:

$\begin{array}{ll}\text { LFC } & \text { FVC } \\ 74 & 4.2 .1 \\ 150 & 4.6 .6 \\ 61 \text { y } 249 & 3.4 .19\end{array}$

Con respecto a la primera correspondencia, Oliva Manso piensa que el capítulo 4.2.1 en el que no hay referencias personales, introduce nuevas consideraciones, además de ampliar y clarificar la norma que recoge el $\mathrm{LFC}^{30}$. Al compararlos, sin embargo, he visto que aunque la regla abstracta de ambas colecciones aborda el mismo problema de partida, es decir qué hacer en caso de que un hombre (A) compre una heredad a otro (B) y haya un tercero (C) que reclame la heredad como suya, cada colección presta atención a una posibilidad diferente: mientras el LFC se centra en las consecuencias jurídicas para $\mathrm{A}$ en caso de que $\mathrm{C}$ gane el pleito, el FVC se interesa por las consecuencias jurídicas para $\mathrm{A}$ en caso de que actúe a espaldas de $\mathrm{B}$. Así pues, ambos pleitos parten de la mima situación, sin embargo, cada uno de ellos evoluciona de manera diferente. El FVC, al igual que el LFC, se decanta por abordar un aspecto muy concreto del juicio, lo que impide concluir que la norma del primero tiene un alcance más general que la del segundo.

Por otro lado, el hecho de que el LFC incorpore el nombre de personas que vivieron esta situación jurídica y el FVC no, no implica que la norma del FVC sea más general. ¿Por qué el FVC no lo hace y el LFC, sí? Las respuestas pueden ser múltiples y diversas. Por ejemplo, se podría pensar que tal decisión está relacionada con el hecho de que al tratarse de juicios diferentes, el redactor del LFC tenía a mano un caso concreto juzgado mediante esta regla, mientras que el redactor del FVC, no; o quizá, como se ha visto hasta ahora, al adoptar el redactor del LFC un tono más didáctico, sintió la necesidad de ilustrar con mayor frecuencia los fueros. En todo caso, la hipótesis de la territorialización no se puede dar por válida con estos ejemplos.

Para Oliva Manso, la regla de 4.6.6 del FVC proviene de la fazaña 150 del LFC y ambos serían el mejor ejemplo de territorialización ${ }^{31}$. No obstante, esta comparación presenta un problema: si bien es verdad que al comparar el contenido de ambos, la norma dada en 4.6.6 se ajusta a la perfección al contenido del pleito descrito en 150, Oliva Manso da por sentado que el capítulo 150 es una fazaña, cuando no es así. Una vez más el redactor del LFC introduce elementos en el pleito que permiten matizar: desde el inicio, deja claro que no estamos ni ante otro fuero anecdótico ni ante una fazaña, sino ante un fuero de vna fasannia. ¿Qué quiere decir esto?, que el redactor del LFC veía ya en el momento de la redacción que esta anécdota era diferente a las otras porque, a partir de este pleito, se generó una norma y de ahí la frase intro-

J. Alvarado Planas y G. Oliva Manso, Los fueros de Castilla, Madrid, 2004, p. 204.

Oliva Manso afirma que este es un ejemplo de territorialización de una fazaña dado por Galo Sánchez, pero esta comparación no figura en el estudio de Sánchez (Ibidem, p. 205). 
ductoria. Esta explicación aparece reforzada por lo que el redactor comenta al final: [...] Et el juysio fue atal que ninguna heredat nueua non deue faser mal ala heredat vieia. Et sy non, deue pechar çien marauedis et el danno doblado. Al incorporar esta aclaración, el redactor no sólo insiste en que se trata de un juicio diferente, además aprovecha para destacar lo que le interesa del pleito: este es importante por las repercusiones jurídicas que tuvo posteriormente. A pesar de esto, Oliva Manso cree que el redactor del FVC sería un redactor mucho más hábil y concienzudo que el redactor del LFC porque supo redactar una norma a partir de un juicio concreto. Al afirmar esto, ignora la voz del redactor del LFC, una voz que nos muestra a alguien que sabía lo que se hacía. Este redactor llevó a cabo una labor tan completa como el redactor de la otra colección, aunque, eso sí, desde otra perspectiva.

Para terminar, Oliva Manso propone que las normas contenidas en los capítulos 61 y 249 del LFC han sido territorializadas por el redactor del FVC al realizar el capítulo 3.4.1932. Ya he analizado las diferencias que hay entre el fuero 61 y la fazaña 249 al hablar del artículo de Galo Sánchez y he mostrado que ambos evocan aspectos diferentes de un conflicto generado a raíz del pago de una deuda. Esto mismo se puede objetar si se compara sus contenidos con el del capítulo 3.4.19. En efecto, la fazaña 249 evoca un problema que surge a partir de una deuda entre un hombre que muere y un hombre judío, mientras que el capítulo 3.4.19 habla de la deuda que adquiere un hombre vivo con un hombre de religión judía. La anécdota de la fazaña no puede considerarse en ningún caso como la versión local a partir de la cual se genera la norma contenida en 3.4.19. Quedaría por hablar de la diferencia de contenidos de los capítulos 61 y 3.4.19, aunque no lo haré dado que mi análisis no se centra en comprender la diferencia entre los fueros de ambas colecciones, sino en comprender el uso de la anécdota en el derecho territorial.

En todo caso, las comparaciones realizadas por Oliva Manso en las que incluye capítulos anecdóticos ni confirman el proceso de territorialización ni permiten comprender mejor las diferencias que se dan entre los capítulos del FVC y el LFC.

\section{Conclusión}

Galo Sánchez fue el primero en analizar la redacción del derecho territorial de Casti1la. Sus conclusiones han marcado la visión de todos aquéllos que se han interesado por el tema posteriormente. Para él, estas colecciones mostrarían un sin fin de defectos, debido a la impericia de sus redactores. Estos no fueron capaces de manejar correctamente las fuentes a la hora de realizar las refundiciones y de ahí que muchos capítulos se repitan e incorporen discurso anecdótico.

Ahora bien, el problema de la hipótesis de Galo Sánchez es que no analiza de manera sistemática ni la anécdota de los fueros ni la de las fazañas, de ahí que no haya tenido en cuenta, por ejemplo, el fuero 1.2.2 del FVC, fuero que contradice su hipótesis sobre la evolución de fazaña a fuero en el derecho territorial castellano. Es imprescindible, por tanto, volver a examinar el uso que se hace de la anécdota en estas colecciones. Solo así se descubre que los redactores la usan con unos fines concretos: cuando se estudia su comportamiento, lo primero que salta a la vista es que

Idem, p. 206. 
los redactores no incluyen repeticiones fatuas, sino que tratan los conflictos desde diferentes perspectivas, intentando ser lo más exhaustivos posible. En segundo lugar, que al elaborar los fueros, estos juzgaron oportuno incluir anécdota para dar mayor legitimidad a las reglas, además de permitirles abordar su contenido de una manera más didáctica. En efecto, los fueros acompañados de referencias concretas resultan más sencillos de comprender, dado que aclaran el contenido de las reglas mediante referencias más cercanas para el auditorio al que iban dirigidas.

En resumidas cuentas, al comparar la labor del redactor del LFC y la del FVC, se observa que ambos manejan el discurso con unas intenciones comunicativas precisas. La técnica de ambos resulta adaptada a los fines que persiguen y desde ningún punto de vista se puede clasificar como rudimentaria, torpe o primitiva.

Por supuesto, para llegar a conclusiones definitivas, es necesario continuar adelante con el análisis de la redacción de los otros capítulos de estas colecciones, sobre todo con el análisis de las fazañas. Quizá de esta manera, se vea por fin que cuando un fuero incorpora anécdota no se debe a que estamos frente a una antigua fazaña.

\section{Bibliografía citada}

Alvarado Planas, Javier, «El proceso de redacción de los fueros de Castilla», en Historia iuris: estudios dedicados al profesor Santos M. Coronas González, Oviedo, 2014, vol. I, pp. 261-278.

Alvarado Planas, Javier y Gonzalo Oliva Manso, Los fueros de Castilla, Madrid, 2004.

Bremond, Claude, Jacques Le Goff y Jean-Claude Schmitt, L'exemplum, Turnhout, 1996.

Corominas, Joan y José Antonio Pascual, Diccionario crítico etimológico castellano e hispánico, Madrid, 1980.

Charaudeau, Patrick y Dominique Maingueneau, Dictionnaire d'analyse du discours, París, 2002.

Domínguez Lozano, Pilar, Las circunstancias personales determinantes de la vinculación con el derecho local. Estudio sobre el derecho altomedieval y el derecho local de Aragón, Navarra y Cataluña (siglos $I X-X V$ ), Madrid, 1988.

García Gallo, Alfonso, «Textos de derecho territorial castellano», AHDE, XIII, 1936-

1941, pp. 308-396.

García Ulecía, Alberto, Los factores de diferenciación entre las personas en los fueros de la Extremadura castellano-aragonesa, Sevilla, 1975.

González Alonso, Benjamín, Ángel Barrios García y Gregorio del Ser Quijano, Fuero viejo de Castilla. Consideraciones sobre la historia del derecho de Castilla (c. 800-1356), Valladolid, 1996.

Iglesia Ferreirós, Aquilino, «Derecho municipal, derecho señorial, derecho regio», Historia, Instituciones, Documentos, IV, 1977, pp. 115-197.

Kabatek, Johannes, Die Bolognesische Renaissance und der Ausbau romanischer Sprachen. Juristische Diskurstraditionen und Sprachentwicklung in Südfrankreich und Spanien im 12. und 13. Jahrhundert, Tübingen, 2005.

Kabatek, Johannes, «Tradiciones discursivas y cambios lingüísticos», Lexis. Revista de Lingüistica y Literatura, XXIX/2, 2005, pp. 151-177.

Malenfant, Marie-Claude, Argumentaires de l'une et l'autre espèce de femme. Le statut de l'exemplum dans les discours littéraires sur la femme (1500-1550),Quebec, 2003. 
Ruiz Gómez, Francisco, Las aldeas castellanas en la Edad Media. Oña en los siglos XIV y $X V$, Madrid, 1990.

Sánchez, Galo, Libro de los fueros de Castiella, Barcelona, [1924] 1981.

Sánchez, Galo, «Para la historia de la redacción del antiguo derecho territorial castellano», AHDE, VI, 1929, pp. 260-328.

Valle Videla, Luz, «Los elementos concejiles en los capítulos anecdóticos del Libro de los fueros de Castiella», en Javier Alvarado Planas, El municipio medieval:

nuevas perspectivas, Madrid, 2009, pp. 201-219.

Valle Videla, Luz, «El discurso referido en los fueros anecdóticos del Libro de los fueros de Castiella», en Luz Valle Videla, El discurso referido en los textos medievales españoles, París, 2013, pp. 157-172.

Valle Videla, Luz, «Antroponimia y representación en el Libro de los fueros de Castiella», en Cesar García de Lucas y Alexandra Oddo, Magister dixit. Mélanges offerts à Bernard Darbord, París, 2016, pp. 419-433. 